\section{Consommation alimentaire}

\section{Les apports en lipides des français sous la loupe de l'Onidol}

L'Anses a mis à disposition en septembre dernier les données brutes de l'étude nationale de consommation alimentaire Inca. L'Onidol, interprofession des oléagineux, a analysé et comparé ces données aux apports nutritionnels conseillés (ANC) pour les acides gras, mettant en évidence d'importants déséquilibres dans l'alimentation des Français.

L'étude révèle ainsi que :

- l'apport moyen en lipides et l'apport en oméga 6 sont satisfaisants ;

- l'apport en oméga 3 est largement inférieur aux ANC : 98,8 \% des Français ont un apport insuffisant en acide alpha-linolénique, alors que cet acide gras est essentiel car le corps humain ne peut pas s'en passer et il ne sait pas le fabriquer;

- l'apport en oméga 9 est lui aussi inférieur aux recommandations;

- à l'inverse, les acides gras saturés sont apportés en quantités trop importantes, en particulier les acides gras saturés spécifiques athérogènes en cas d'excès.

Pour en savoir plus : communiqué de presse ONIDOL et rapport complet, février 2015.

\section{Carrière}

\section{Nouvel interlocuteur au Département Analyse \& Expertise de l'lterg}

Loïc Leitner a rejoint le département Analyse \& Expertise de l'Iterg en tant que chargé d'affaires ; il assurera la continuité du poste tenu par Jean-Louis Coustille qui a pris sa retraite en avril 2015. Docteur en génie des procédés et des produits, il occupait un poste d'ingénieur de recherche à l'INSA (Institut National des Sciences Appliquées) de Toulouse avant d'intégrer l'Iterg.

\section{Internet}

\section{Le GIE Matinnol a désormais son site}

Le GIE Matinnol (MATuration des INNovations en OLéochimie), qui accompagne les industriels pour le développement de procédés visant à convertir les lipides et dérivés lipidiques en produits chimiques renouvelables, a ouvert son site Internet www.matinnol.eu.
Rappelons que le GIE Matinnol a été créé par l'Iterg et la SAS Pivert pour offrir aux industriels un guichet unique vers les plates-formes d'innovation Cedop et Biogis center.

La direction de ce GIE est assurée par Carine Alfos, directrice Innovation à l'Iterg.

\section{$3^{\text {e }}$ Conférence mondiale sur l'agriculture climato-intelligente}

\section{Répondre aux enjeux du changement climatique}

À l'occasion de la $3^{\mathrm{e}}$ Conférence mondiale sur l'agriculture climato-intelligente qui s'est tenue à Montpellier du 16 au 18 mars 2015, plus de 700 participants venant de 75 pays à travers le monde se sont réunis pour faire le point sur l'état des connaissances et s'accorder sur une stratégie de recherche globale et locale. La conférence a rassemblé des représentants d'organisations scientifiques, d'organisations gouvernementales nationales et internationales, des associations d'agriculteurs, des industriels, des ONG et la société civile.

Aujourd'hui, l'agriculture est face à un défi. Le changement climatique impacte déjà négativement la production alimentaire alors que la demande va très fortement évoluer au cours des 40 prochaines années. De plus, l'agriculture doit réduire ses émissions de gaz à effet de serre qui sont largement associées aux processus biologiques lors de la production agricole. Faute d'atteindre ces objectifs, la sécurité alimentaire et la stabilité du climat ne pourront être atteints.

Le concept d'agriculture climato-intelligente a été lancé il y a 5 ans dans le but de mobiliser les scientifiques et autres parties prenantes pour trouver des solutions d'adaptation et d'atténuation du changement climatique, répondre aux défis de la sécurité alimentaire et anticiper les problématiques à venir. Partant du constat que le changement climatique impacte déjà sérieusement l'agriculture et affectera particulièrement les agriculteurs et les pays les plus fragiles, les scientifiques alertent sur la nécessité de mobiliser toutes les connaissances afin d'agir maintenant pour préparer l'avenir. Ils témoignent de l'urgence d'identifier les questions clés des 20 à 30 prochaines années et qui nécessitent un investissement de recherche et de prospective dès à présent.

Les représentants de la conférence soulignent également que des solutions qu'offre l'agriculture climato-intelligente existent déjà et peuvent être mises en œuvre à condition qu'il y ait un réel engagement des acteurs concernés. De plus, les transitions agricoles agiront comme l'un des principaux leviers dans d'autres secteurs, notamment l'emploi, l'énergie, l'agroalimentaire, la santé, l'alimentation et l'environnement. 
Lors de la conférence, Stéphane Le Foll, ministre de l'Agriculture, de l'Agroalimentaire et de la Forêt, a annoncé que des fonds seront mobilisés pour un programme international de recherche sur la restauration des sols dégradés et le stockage de carbone, enjeux majeurs qui s'inscrivent dans les trois piliers de l'agriculture climato-intelligente (adaptation, atténuation, sécurité alimentaire).

Les négociations sur le climat intègrent progressivement le potentiel de transition en agriculture. Grâce à l'agriculture climato-intelligente, la communauté scientifique peut s'engager au-delà des frontières et développer une interaction avec les acteurs du monde agricole et les politiques, et promouvoir une diplomatie scientifique.

La science peut en effet changer la perception d'un secteur agricole responsable du changement climatique en démontrant qu'il est aussi vecteur de solutions. Le caractère prioritaire de la transition agricole peut constituer une première étape pour une action politique sur le changement climatique, et particulièrement à la COP21 à Paris.

La $3^{e}$ Conférence scientifique mondiale sur l'agriculture climato-intelligente était organisée par le Cirad, l'Inra, l'IRD et Agropolis International avec le soutien du CGIAR, de l'Université de Wageningen, de l'Université de Davis, de la FAO et du GFAR. La conférence a bénéficié du soutien financier du ministère des Affaires étrangères et du Développement international et du ministère de l'Agriculture, de l'Agroalimentaire et de la Forêt, de la Région Languedoc-Roussillon, de Montpellier Méditerranée Métropole, des LabEx Agro et CeMEB.

\section{Label \& Agrément}

\section{Renouvellement du label CRT de l'Iterg}

L'Iterg a obtenu le renouvellement de son label CRT (Centre de Ressources Technologiques) pour une durée de 3 ans, 2015-2017. Pour rappel, la qualification de CRT est attribuée aux structures capables de répondre avec professionnalisme aux besoins des PME/PMI. Un cahier des charges, établi avec l'AFNOR, décrit les conditions minimales requises pour obtenir ce «label qualité ». Trois caractéristiques des CRT sont décrites comme essentielles :

- professionnalisme dans l'exécution des prestations réalisées, en particulier en termes de résultats, coût, délai et confidentialité ;

- réalisation de prestations sur mesure pour les entreprises;

- les prestations doivent concerner des projets innovants des PME-PME.

Il s'agit de transfert de technologie de la recherche vers les entreprises. Ceci implique un contact permanent avec des laboratoires de recherche académique.

\section{Le laboratoire d'analyses physico-chimiques de l'Iterg agréé par le COI}

Le laboratoire d'analyses physico-chimiques de l'Iterg a obtenu à nouveau l'agrément du Conseil oléicole international
(COI) pour la période du $1^{\text {er }}$ décembre 2014 au 30 novembre 2015. Le jury de dégustation d'huile d'olive vierge a également obtenu l'agrément pour la même période. Les listes des laboratoires agréés sont disponibles sur le site du COI.

\section{Formation}

\section{Potentiel des sourcings lipidiques}

L'Iterg organise le mardi 23 juin 2015 à Pessac (33) une journée d'information dédiée aux nouveaux sourcings lipidiques : oléagineux, microorganismes, microalgues, insectes.

Au programme :

9 h 30 : Accompagnement Iterg au développement nouveaux sourcings - identification des verrous à la mise sur le marché. Carine Alfos, Iterg

10 h 10 : Production de lipides d'intérêt chez la levure, par Carole Molina-Jouve, Critt Bio-Industries

10 h 50 : Bioraffinerie d'insectes (Ento-raffinerie), par Antoine Hubert, Ynsect

11 h 30 : La bio-production de lipides grâce aux microalgues, par Pierre Calleja, Fermentalg

12 h 30 : Pause déjeuner

14 h 00 : Adaptation des agroressources oléagineuses, par Frédéric Fine, Cetiom

14 h 40 : Omega-9 Oils la nouvelle génération, par Benjamin Jeunink, Dow

15 h 20-16 h : Échanges

Inscription en ligne obligatoire $(100 €$ TTC par personne pour les entreprises non adhérentes, gratuit pour les adhérents à l'Iterg) avant le 2 juin 2015 sur : http://iterg.com/form/

\section{Vient de paraître}

\section{Évaluation sensorielle, guide des bonnes pratiques}

L'évaluation sensorielle permet d'examiner les propriétés organoleptiques d'un produit. Différentes méthodes existent : essais analytiques pour décrire les produits et hédoniques pour mesurer leurs côtés plaisants ou déplaisants. Ces principes de base sont continuellement enrichis de nouveaux développements qui nécessitaient la mise à jour du guide Actia publié en 1999.

Cette version réactualisée par les partenaires du RMT Actia Sensorialis a pour objectif de mettre à la portée des usagers de l'évaluation sensorielle un document complet, dégageant l'essentiel à retenir des différentes approches, validé par des experts, tout en étant attractif et de lecture facile. L'accent est mis sur les éléments à respecter pour apporter les garanties de bonnes pratiques dans le domaine de l'évaluation sensorielle.

Parmi les nouveautés : les essais de positionnement global, la dynamique temporelle des perceptions, la taille du groupe 
dans le cadre des tests hédoniques, la mise en relation des essais analytiques et hédoniques...

Évaluation sensorielle, guide des bonnes pratiques. Edition 2014. Actia.

Auteurs : Hélène Albouy (ÉnilV), Françoise Béhérec (Actalia), Jean-Pierre Bodin (Énilia Ensmic), Marie-Hélène Desmonts (Aérial), Dominique Droger (Actalia), François Germon (Agrotec), Virginie Herbreteau (Actalia), Sylvie Issanchou (Inra-CSGA), Karen Joly (Welience), Pascal Schlich (Inra-CSGA)

Coordination : Alice Dulas (Actia), Virginie Herbreteau (Actalia)

Préface par Alexandre Voirin (Nestlé) et Sylvie Issanchou (Inra-CSGA)

Auteurs $1^{\text {re }}$ édition : Didier Majou (Actia) coordination; Marie-Pierre Béague, Françoise Siret (CTSCCV), Françoise Besnier, Sylvie Sibiril (Adria), Colette Breysse (Casimir), Élisabeth Catherine (SSHA), Jean-François Clément (ActilaitLes maisons du goût), Marie-Hélène Desmonts (Aérial), Jean-Luc Herbommez (Cerbia), Marie-Pierre Jacquin (C.A3), Laurent Lebœuf (IFBM), Dominique Lepais (Agrotec), JeanChristophe Lombard (CTCPA), Renée Raoux (Iterg), Frank Tonon (Agro-Hall), Frédéric Vey (Adria).

Rédacteurs : Laurent Aron, Clarisse Broussard. (Inra).

\section{Changement climatique : impacts et adaptations}

L'étendue des travaux scientifiques menés par les acteurs du Languedoc-Roussillon sur l'impact ou l'adaptation au changement climatique est très vaste. Loin d'être exhaustif, le numéro 20 des Dossiers d'Agropolis International a pour ambition d'offrir au lecteur un panorama de ces travaux, en présentant les acteurs impliqués et quelques exemples concrets de leurs activités de recherche.

Au sommaire de ce dossier : Changement climatique \& interactions entre organismes; Thématiques couvertes par les structures de recherche; Changement climatique \& ressources, territoires et développement; Changement climatique \& biodiversité et écosystèmes ; Biodiversité et écosystèmes continentaux ; Biodiversité et écosystèmes marins ; Changement climatique \& systèmes de production agricole et d'élevage.

Changement climatique : impacts et adaptations - Les dossiers d'Agropolis International - $\mathrm{N}^{\circ}$ 20, février 2015. Coordination scientifique : Sandra Ardoin-Bardin (IRD), Nicolas Arnaud (CNRS), Sophie Boutin (UM), Jean-Luc Chotte (IRD), Philippe Jarne (CNRS), Pascal Kosuth (Agropolis Fondation), Philippe Lebaron (UPMC), Éric Servat (IRD). Coordination Agropolis International : Mélanie Broin

Le dossier est téléchargeable sur le site d'Agropolis http:// www.agropolis.fr/ Accueil > Publications > Les « Dossiers d'Agropolis International». 